\title{
Efeitos gradativos do to@ue terapêutico na redução da ansiedade de estudantes universitários
}

Gradual effects of therapeutic touch in reducing anxiety in university students

Efecto gradual del tacto terapéutico en la reducción de la ansiedad en estudiantes universitarios

Vanessa Miranda Gomes', Maria Júlia Paes da Silva', Eutália Aparecida Cândido Araújo'

'Universidade de São Paulo. Escola de Enfermagem, Departamento de Enfermagem Médico-Cirúrgica. São Paulo, SP

Submissão: 23/06/2007

Aprovação: 30/10/2008

\section{RESUMO}

Trabalho de pesquisa Quantitativo realizado com 42 discentes de uma instituição pública de ensino superior, utilizando-se a técnica do Toque Terapêutico - Método Krieger-Kunz e aplicação de um Questionário validado no Brasil para avaliar a ansiedade durante três sessões. Os sujeitos foram alocados em dois grupos: experimental (1), em Que receberam a terapia complementar; e controle (2), em Que foram submetidos a imitação da técnica sem intenção terapêutica. O objetivo foi identificar a influência gradativa desta terapia complementar de saúde no Que se refere ao estado de ansiedade dos estudantes. A análise dos dados revelou uma redução estatisticamente significativa do estado de ansiedade em ambos os grupos, com pd" 0,05.

Descritores: Toque terapêutico; Ansiedade; Terapias alternativas; Estudantes de enfermagem.

\section{ABSTRACT}

This is Quantitative research conducted with 42 students of a public university using the Therapeutic Touch - Krieger-Kunz Method and the application of a Questionnaire validated in Brazil to assess anxiety in three sessions. Subjects were divided into two groups: experimental (1), in which the complementary therapy was used; and control (2), in which a mock of the technique, with no therapeutic intention, was applied. The objective was to identify the gradual influence of that health complementary therapy upon the students' state of anxiety. The analysis of the data showed a statistically significant reduction of the state of anxiety in both groups, with pd" 0.05 .

Descriptors: Therapeutic touch; Anxiety; Alternative therapies; Nursing students.

\section{RESUMEN}

Trabajo de pesquisa cuantitativo con 42 discentes de una institución pública de enseñanza superior, utilizándose la técnica del Toque Terapéutico - Método Krieger-Kunz y aplicación de un cuestionario validado en Brasil para evaluar la ansiedad durante tres sesiones. Los sujetos fueron alocados en dos grupos: experimental ( I) Que recibieron la terapia complementar y control (2) Que fueron sometidos a la imitación de la técnica sin intención terapéutica. El objetivo fue identificar la influencia graduada de esta terapia complementar de salud en lo Que se refiere al Estado de Ansiedad de los estudiantes. La análisis de los datos rebeló una reducción estadísticamente significativa del Estado de ansiedad en ambos los grupos con pd" 0,05.

Descriptores: Tacto terapéutico; Ansiedad; Terapias alternativas; Estudiantes de enfermería. 


\section{INTRODUÇÃO}

A Enfermagem holística tem se inserido no campo da ciência como um paradigma do pensar humano, e o desenvolvimento de teorias de Enfermagem, como A Ciência do Ser Humano Unitário(1), fortalece as práticas holísticas de Enfermagem. Segundo Martha Rogers, o ser humano está integrado no todo e o todo está integrado no ser humano, mas cada ser humano tem um padrão próprio (individual).

O Toque Terapêutico (TT) é uma técnica de terapia complementar Que não possui QualQuer base religiosa e cujo intuito é repadronizar o campo energético humano ${ }^{(2-4)}$. Tem se revelado um excelente meio não-invasivo de Que o enfermeiro e outros profissionais da saúde lançam mão para promover relaxamento, reduzir a ansiedade, controlar a dor e outros efeitos ${ }^{(2,5,6)}$.

Embora o Toque Terapêutico represente uma nova técnica no nosso atual sistema de cuidados à saúde, ele se originou da prática milenar da imposição de mãos ${ }^{(3,4)}$, e consiste na prática de harmonização do campo energético humano $(\mathrm{CEH})$ através do uso consciente das mãos, com o intuito de restabelecer pessoas enfermas. Foi desenvolvido e sistematizado como método de terapêutica complementar de Enfermagem pela enfermeira Dra Dolores Krieger, em 1972, na Universidade de Nova York ${ }^{(4)}$.

Com a inclusão do Diagnóstico de Enfermagem "campo de energia perturbado" pela North American Nursing Diagnosis Association $^{(7)}$, o Toque Terapêutico foi reconhecido como uma prática complementar de Enfermagem e pode ser utilizado diariamente na assistência aos pacientes. No entanto, percebemos Que no Brasil ainda são poucos os profissionais Que dominam esta técnica e, por isso esse importante instrumento do cuidado vem sendo pouco utilizado nos hospitais.

Cabe ressaltar Que o Conselho Federal de Enfermagem na Resolução COFEN-197 de 19 de março de 1997, estabelece e reconhece as Terapias Alternativas como especialidade e/ou Qualificação do Profissional de Enfermagem ${ }^{(8)}$. É preciso enfatizar Que o Toque Terapêutico, assim como todas as Terapias Complementares, é um tratamento auxiliar, Que não dispensa o tratamento convencional e pode ser realizado paralelamente às demais medidas implementadas pela equipe de saúde.

Refletindo sobre o contexto no Qual a Enfermagem vem desenvolvendo o seu trabalho, onde os conhecimentos científicos e novas tecnologias já pouco atendem às necessidades das pessoas Que precisam de cuidados, parece-nos ser relevante o profissional enfermeiro conhecer e desenvolver habilidades na utilização dos instrumentos do cuidado de Enfermagem relacionados à cidadania, à busca de direitos, à Qualidade dos serviços e resgate do cuidado com uma visão complexa e humanista ${ }^{(9)}$.

A aplicação do Toque Terapêutico método Krieger-Kunz pode colaborar efetivamente para uma melhor Qualidade de vida, além de a técnica não utilizar alta tecnologia e ser de baixo custo. Acredita-se que a Ciência da Enfermagem têm muitas possibilidades de investigações, principalmente em relação à aplicação de Terapias Complementares, Que possam trazer benefícios à humanidade.

\section{OBJETIVO}

Verificar se a utilização do Toque Terapêutico - Método Krieger-
Kunz produz alterações gradativas no Estado de Ansiedade dos estudantes após três sessões.

\section{MÉTODO}

\section{Tipo de Estudo}

Foi realizado um estudo de campo experimental longitudinal com abordagem Quantitativa, no Qual os sujeitos da pesquisa foram alocados aleatoriamente em dois grupos: Experimental (1) e Controle (2) tendo, portanto, cada sujeito a mesma probabilidade de ser encaminhado a Qualeuer um dos grupos.

\section{Campo de Estudo}

A coleta de dados foi realizada na Escola de Enfermagem da Universidade de São Paulo - EEUSP, instituição de caráter público, localizada na cidade de São Paulo.

\section{Sujeitos e Critérios de Inclusão}

A amostra de 42 alunos foi composta de universitários da EEUSP Que se propuseram participar. A inclusão dos sujeitos na pesQuisa foi feita após a assinatura do Termo de Consentimento Livre e Esclarescido, conforme previsto pela Resolução CNS 196/1996, Que fornece as diretrizes e normas regimentais de pesquisa, envolvendo seres humanos.

\section{Instrumentos}

Para a coleta de dados foi utilizado o Inventário de Ansiedade Traço-Estado, IDATE ${ }^{(10)}$, já validado no Brasil.

\section{Procedimentos de Coleta de Dados}

Após aprovação do CEP da EEUSP (Processo no 630/2007/ CEP-EEUSP), os alunos foram contatados por uma das autoras em sala de aula, e os Que concordaram em participar, além de assinarem um Termo de Consentimento Livre e Esclarecido, responderam o Inventário de Ansiedade Traço. Foram informados que apenas metade deles receberia o Toque Terapêutico Método Krieger-Kunz, e Que a outra metade seria abordada com movimentos semelhantes sem nenhuma intenção terapêutica. Para alocação aleatória dos sujeitos nos grupos de estudo, experimental (1) e controle(2), foi realizado um sorteio prévio por uma das autoras. Em seguida, foram agendados os atendimentos individuais com três sessões, com intervalos de dois dias. Imediatamente antes e após as sessões os alunos responderam ao Inventário de Ansiedade Estado ${ }^{(10)}$ para comparação dos resultados nos dois grupos de estudo Experimental (1) e Controle (2). Os alunos foram informados que a autora Que coletou os dados fez o curso de Toque Terapêutico Método KriegerKunz para iniciantes, ministrado por enfermeira, doutora na área de T.T., no Instituto Lin Pin Chuan.

\section{Análise dos Dados}

Foi solicitado o auxílio de uma bioestatística para aplicação de testes paramétricos Que permitiram avaliar a significância dos dados. Neste trabalho, o nível de significância adotado foi de 5\%. As estatísticas com p descritivo d" 0,05 são consideradas significantes.

A técnica estatística empregada foi o GLM para medidas repetidas (modelo linear generalizado) Que é um procedimento onde se incorpora variáveis dependentes normalmente distribuídas 
e variáveis independentes, categóricas (fatores) ou contínuas (covariáveis), para se verificar a ocorrência ou não de diferenças entre antes e depois nas avaliações, considerando os dois grupos em separado. Para se verificar se os grupos diferem entre si ou não, empregaram-se os testes múltiplos de comparação 2 a 2 de Bonferroni ajustado. Empregaram-se, também, o teste $t$ independente para comparar o estado de ansiedade antes e estado de ansiedade depois, segundo os grupos, nas três sessões, e o teste t pareado para comparar o traço de ansiedade com o estado de ansiedade antes, também nas três sessões.

\section{RESULTADOS}

\section{Caracterização da Amostra}

Do total de estudantes Que participaram da coleta (42), 100\% responderam os instrumentos corretamente, selecionando uma alternativa em todos os campos dos instrumentos; portanto, para análise dos dados foram considerados 42 sujeitos neste estudo, sendo Que 21 (50,0\%) sujeitos compõem o grupo experimental (1) e 2 I (50,0\%) o grupo controle (2).

Com base nos dados obtidos, observa-se Que o sexo feminino foi predominante com $97,6 \%$, fato esperado, visto o estudo ser realizado com os alunos de graduação em Enfermagem, Que tradicionalmente é um curso com maioria feminina. A faixa etária predominante foi de 23 a 24 anos (30,9\%). Quanto ao semestre Que estavam cursando na graduação, a maioria dos estudantes $(47,6 \%)$ cursava o sexto semestre.

\section{Características Relacionadas à Ansiedade dos Sujeitos}

Com o IDATE traço pode-se classificar os níveis de ansiedade que os sujeitos da pesquisa apresentaram como parte de sua personalidade. A figura I mostra a característica de ansiedade permanente, mencionada com base nas respostas do inventario de Ansiedade Traço distribuída pelos grupos experimental (1) e controle (2).
Com base nos dados apresentados na figura 1, identifica-se Que o Traço de ansiedade predominante é o moderado, seguido pelos níveis elevado, baixo e altíssimo respectivamente, independente do grupo analisado.

Para comparar o Traço de ansiedade entre os grupos foi utilizado o Teste $t$ independente, e não se observaram diferenças estatisticamente significativas entre os grupos, Quanto à característica de ansiedade $(p=0,629)$.

\section{Comparação entre as medidas realizadas antes e depois das sessões de Toque Terapêutico}

O nível de ansiedade antes e após cada sessão de Toque Terapêutico foi medido com o inventário de ansiedade estado (IDATE). A figura 2 mostra a característica de ansiedade transitória antes e depois da primeira sessão de Toque Terapêutico distribuída pelos grupos experimental (1) e controle (2), mencionada com base nas respostas do inventario de Ansiedade Estado.

Antes da primeira sessão de Toque Terapêutico, o grupo experimental (1) apresentou-se com $61,9 \%$ dos sujeitos com nível de ansiedade estado moderado, 33,3\% elevado e 4,8\% baixo. $\mathrm{O}$ grupo controle (2) apresentou antes da técnica, na primeira sessão, 71,4\% dos sujeitos com nível moderado de ansiedade estado, seguido pelo nível de ansiedade elevado com 19,0\% e baixo com 9,5\% dos sujeitos.

Após a primeira sessão, 52,4\% dos sujeitos do grupo 1 apresentou nível de ansiedade estado moderado, o Que significa uma redução do nível de ansiedade, nesta categoria, de 9,5\%, eneuanto Que no grupo 2 a mesma característica foi apresentada por $66,7 \%$ dos sujeitos, com uma Queda de $4,7 \%$; o nível elevado de ansiedade estado foi apresentado por $4,8 \%$ no grupo I, caracterizando uma redução de $28,5 \%$ neste escore de ansiedade, o grupo 2 também apresentou após a primeira sessão 4,8\% dos sujeitos da pesquisa com nível de ansiedade elevado, porém no grupo 2 a redução deste escore foi de apenas 14,2\%; já o nível de

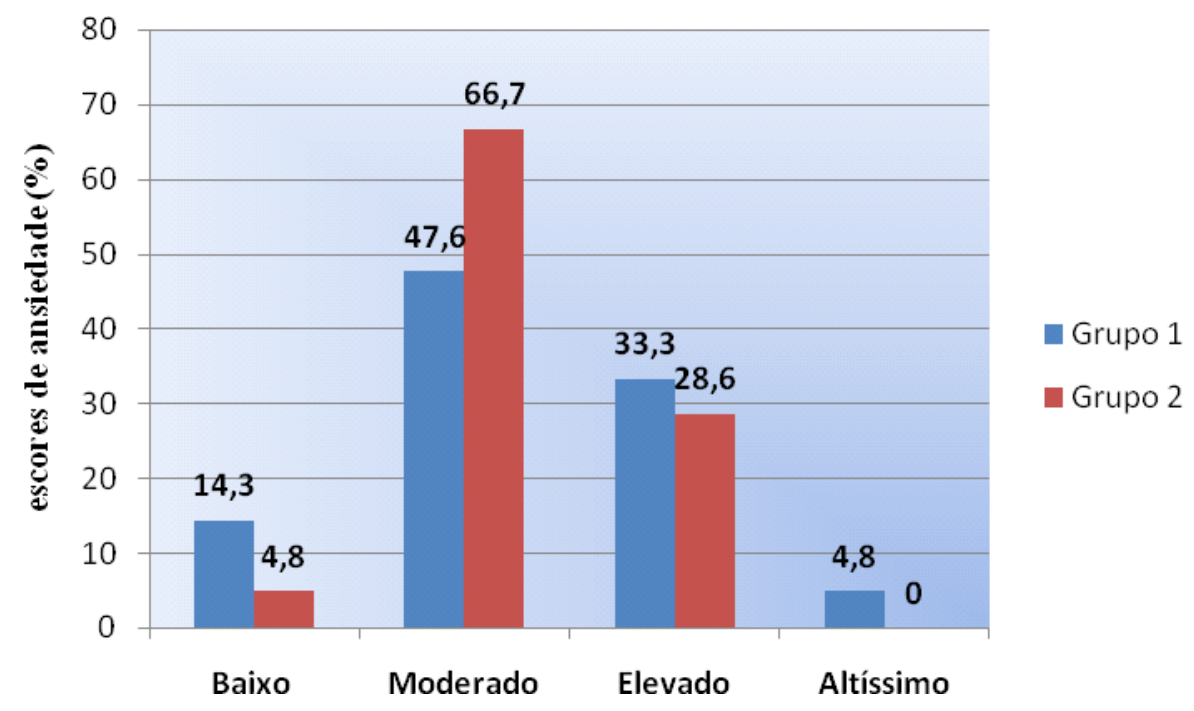

Figura 1. Traço de ansiedade em ambos os grupos: experimental (1) e controle (2). São Paulo, 2007. 


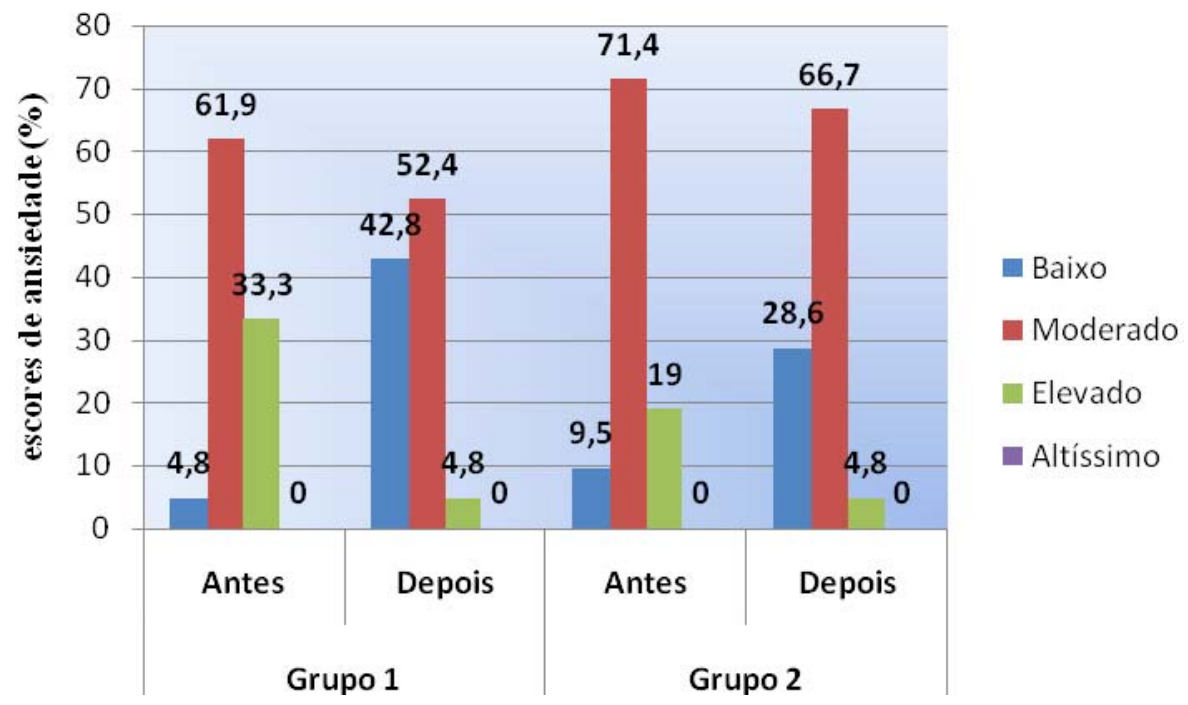

Figura 2. Estado de ansiedade antes e depois do ToQue Terapêutico na primeira sessão distribuída pelos grupos experimental (1) e controle (2). São Paulo, 2007.

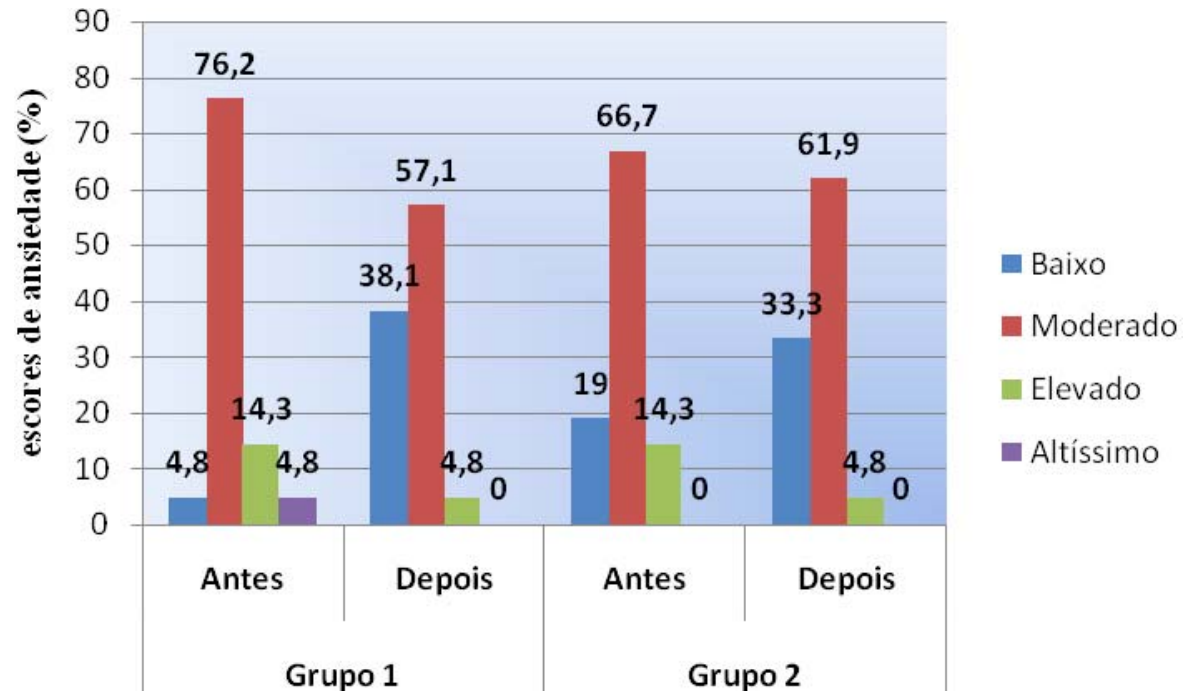

Figura 3. Estado de ansiedade antes e depois do Toque Terapêtico na segunda sessão distribuída pelos grupos experimental (1) e controle (2). São Paulo, 2007.

ansiedade estado baixo após a sessão foi apresentado por $42,8 \%$ dos sujeitos do grupo 1, o Que significa um aumento de $38 \%$ neste escore, no grupo 2 o estado de ansiedade baixo foi apresentado por $28,6 \%$, o Que também resulta em aumento de 19, 1\% de sujeitos nesta categoria.

Para comparar o Estado de Ansiedade antes e depois de cada sessão foi utilizado o teste GLM para medidas repetidas, conforme já referido, e. em ambos os grupos houve uma redução estatisticamente significativa do nível de ansiedade $(p<0,001)$.

O grupo I apresentou uma redução maior do nível de Ansiedade Estado moderado de 4,8\% em relação ao grupo 2, fato Que também se repetiu Quanto ao nível de ansiedade Estado elevado, Quando no grupo experimental (1) a Queda foi de $14,3 \%$ maior do Que no grupo controle (2); já no Que se refere Estado de Ansiedade baixo, houve um aumento de $15,3 \%$ a mais no grupo 1 em relação ao grupo 2 de sujeitos neste escore de ansiedade.

Para comparar o Estado de Ansiedade antes e depois de cada sessão entre os grupos, utilizando-se o Teste $t$ independente, percebeu-se Que na primeira sessão o estado de ansiedade antes e depois não se diferencia entre os grupos, experimental ( I) e controle (2), de modo significativo, com $p=0,243$ e $p=0,249$ respectivamente.

A figura 3 mostra a característica de ansiedade transitória antes e depois da segunda sessão de Toque Terapêutico distribuída pelos grupos experimental (1) e controle (2), mencionada com base nas respostas do inventário de Ansiedade Estado. 


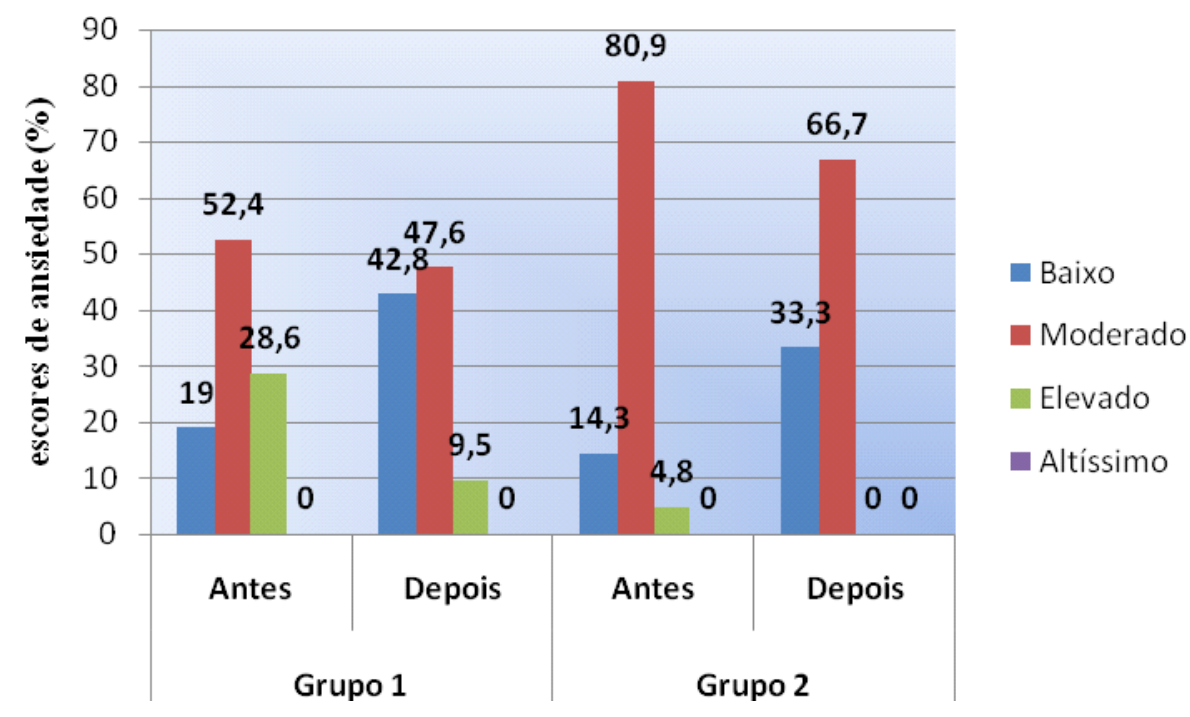

Figura 4. Estado de ansiedade antes e depois do Toque Terapêutico na terceira sessão
distribuída pelos grupos experimental (1) e controle (2). São Paulo, 2007.

O grupo I apresentou-se com 76,2\% dos sujeitos com nível de ansiedade estado moderado antes da segunda sessão de Toque Terapêutico, 14,3\% elevado, 4,8 altíssimo e 4,8\% baixo. O grupo 2 apresentou antes da técnica, na segunda sessão, 66,7\% dos sujeitos com nível moderado de ansiedade estado, seguido pelo nível de ansiedade baixo com 19,0\% e elevado com 14,3\% dos sujeitos.

Depois da segunda sessão o grupo I apresentou-se com 57, 1\% dos sujeitos com nível de ansiedade estado moderado, 4,8\% elevado, $0 \%$ altíssimo e 38, $1 \%$ baixo. O grupo 2 apresentou depois da técnica, na segunda sessão, $61,9 \%$ dos sujeitos com nível moderado de ansiedade estado, 4,8\% elevado e 33,3\% com baixo nível de ansiedade.

Através da utilização do teste GLM para medidas repetidas constatou-se Que houve uma redução estatisticamente significativa do nível de ansiedade ( $<<0,001$ ) Quando comparado o estado de ansiedade antes e o estado de ansiedade depois em ambos os grupos.

O grupo experimental (1) apresentou uma redução total de $66,7 \%$ dos escores de ansiedade após a segunda sessão, sendo esta redução distribuída em nível moderado de ansiedade com Queda de 19,1\%, nível elevado de ansiedade com Queda de 9,5\%, nível altíssimo de ansiedade com Queda de $4,8 \%$, e por fim, houve um aumento de 33,3\% dos sujeitos com nível de ansiedade baixo. O grupo controle (2) apresentou uma redução total de 28,6\% dos escores de ansiedade após a segunda sessão, sendo esta redução distribuída em nível moderado de ansiedade com Queda de 4,8\%, nível elevado de ansiedade com Queda de 9,5\%, e nível de ansiedade baixo com um aumento de $14,3 \%$.

Comparando-se os dois grupos na segunda sessão percebe-se Que houve uma redução maior do estado de ansiedade no grupo experimental, com $38,1 \%$ a mais de Queda dos escores de ansiedade do Que no grupo controle (2).

Na segunda sessão, após a utilização do Teste t independente, observou-se Que o estado de ansiedade antes não se diferencia entre os grupos de modo significativo $(p=0,161)$ e o estado de ansiedade depois também não se diferencia entre os grupos de modo significativo $(p=0,517)$.

A figura 4 mostra a característica de ansiedade transitória antes e depois da terceira sessão de Toque Terapêutico distribuída pelos grupos experimental (1) e controle (2), mencionada com base nas respostas do inventário de Ansiedade Estado.

Na terceira e última sessão, o grupo experimental ( 1 ) apresentou antes da aplicação da técnica $52,4 \%$ dos sujeitos com nível de ansiedade moderado, seguido por $28,6 \%$ com escores elevados de ansiedade e 19,0\% com baixo nível de ansiedade. Após a técnica houve uma Queda de $4,8 \%$ de sujeitos com moderado nível de ansiedade $(47,6 \%)$, redução de $19,1 \%$ no escore elevado de ansiedade $(9,5 \%)$ e aumento de $23,8 \%$ no escore de ansiedade baixo Que passou a apresentar 42,8\%. Pode-se considerar Que a redução da ansiedade ocorreu em 47,7\% dos sujeitos incluídos no grupo experimental (1) após analisar o antes e o depois na terceira sessão.

Após a aplicação do teste GLM para medidas repetidas, observou-se Que houve uma redução estatisticamente significativa do nível de ansiedade antes e após a terceira sessão $(p<0,001)$.

O grupo controle (2) apresentou na última sessão $80,9 \%$ dos sujeitos com nível de ansiedade moderado, seguido por $14,3 \%$ com baixo nível de ansiedade e $4,8 \%$ elevado. Após a técnica mostrou-se com $66,7 \%$ no escore moderado e $33,3 \%$ no escore de ansiedade baixo. Houve uma redução de 14,2\% do nível de ansiedade moderado, 4,8\% no elevado e um aumento de $19 \%$ dos sujeitos com escores de ansiedade baixos; no total ocorreu uma Queda de 38\% do nível de ansiedade após a última sessão.

Ao comparar o grupo I com o grupo 2 na terceira sessão podese afirmar Que o grupo experimental (1) apresentou uma maior redução do nível de ansiedade com $9,7 \%$ a mais de redução do Que o grupo controle (2).

$\mathrm{O}$ Teste $\mathrm{t}$ independente mostra Que não há diferença estatisticamente significativa entre os grupos I e 2 antes e após a terceira sessão, o estado de ansiedade antes não se diferencia entre 
os grupos de modo significativo $(p=0,146)$, e o estado de ansiedade depois também não se diferencia entre os grupos de modo significativo $(p=0,807)$.

Não se observaram diferenças estatisticamente significativas entre os grupos, Quanto ao Traço de ansiedade $(p=0,629)$, porém houve a redução estatisticamente significativa do Estado de ansiedade em ambos os grupos (pd" 0,05).

\section{DISCUSSÃO}

Tendo em vista os dados obtidos com a aplicação do Questionário IDATE-Traço considera-se Que a ansiedade é um problema prevalente entre os alunos da graduação da EEUSP, sendo assim é pertinente empregar esforços na investigação de maneiras de cuidar em Enfermagem no sentido de melhorar a Qualidade de vida do estudante desta Universidade.

Um estudo realizado em 2001 mostra Que para os profissionais, o cuidado de Enfermagem prende-se à satisfação das necessidades dos pacientes com vistas ao seu bem-estar físico e emocional, entretanto, mesmo considerando o relacionamento interpessoal com os seres cuidados, eles se prendem à instância técnica. Já os pacientes aproximam o cuidado de Enfermagem das manifestações de atenção, carinho e paciência, associando-o à afetividade própria do estar-com humano ${ }^{(1)}$. Pode-se inferir, portanto, Que a melhora do Estado de Ansiedade no grupo controle (2), tenha ocorrido pela relação de proximidade entre a terapeuta e os estudantes.

Apesar de não termos encontrado diferenças estatisticamente significativas entre os dois grupos, percebe-se Que a intervenção (do cuidar) reduziu o estado de ansiedade dos estudantes. São muitas as pesquisas, conforme relatado na introdução, Que demonstram a eficácia do $\mathrm{TT}^{(2-6)}$. O número de alunos atendidos e a relação afetiva existente para os dois grupos, pode ter aproximado o resultado entre os grupos.

Mesmo não sendo o objetivo da pes@uisa, é importante registrar Que os estudantes agradeciam muito no final das sessões, demonstrando alegria por estarem sendo cuidados. Vários deles referiram pretender se desenvolver nessa técnica; alguns deles, realmente, já tendo procurado sua capacitação.

\section{CONCLUSÃO E CONSIDERAÇÕES FINAIS}

Consideramos os resultados favoráveis a aplicação do Toque Terapêutico Método Krieger-Kunz, pois existe uma tendência a maior redução dos níveis de ansiedade no grupo em Que foi aplicada a técnica, devido os percentuais de redução nos escores de ansiedade terem demonstrado, nas três sessões, vantagens do grupo 1 em relação ao grupo 2. Sabemos também Que a existência do efeito placebo contribuiu para o resultado significativo apresentado pelo grupo controle (2). Acreditamos Que o nosso cuidado como enfermeiros reflete aquilo Que somos e pensamos, e os nossos clientes sempre irão refletir o que somos como seus cuidadores.

Os resultados obtidos nos estimulam a desenvolver mais pesquisas na temática, Que além de não ser muito invasiva é de baixo custo e... reduz a ansiedade.

\section{REFERÊNCIAS}

I. Rogers ME. An introduction to the theoretical basis of nursing. Philadelphia: Davis; 1970.

2. Sá AC. Aplicação do toque terapêutico em mulheres portadoras do câncer de mama sob tratamento Quimioterápico [tese]. São Paulo (SP): Escola de Enfermagem de São Paulo, Universidade de São Paulo; 2001 .

3. Macrae I. Therapeuthic touch: a practical guide. New York: Alfred A. Knopf; 1987.

4. Krieger D. O Toque Terapêutico. São Paulo: Cultrix; 1996.

5. Marta ISR. Utilização do Toque Terapêutico no processo de cuidar de pessoas com dor crônica não-neoplásica: avaliação da experiência pelo paciente e verificação dos efeitos sobre a intensidade e o significado psicológico da dor e sobre a depressão [tese]. Ribeirão Preto (SP): Escola de Enfermagem de Ribeirão Preto, Universidade de São Paulo; 2000.

6. Movaffaghi Z, Hasanpoor M, Farsi M, Hooshmand P, Abrishami F. Effects of therapeutic touch on blood hemoglobin and hematocrit level. J Holist Nurs 2006; 24(I):4 I-8.

7. North American Nursing Diagnosis Association (NANDA), Diagnósticos de enfermagem da NANDA : definições e classificåção 2001-2002. Porto Alegre: Artmed; 2002.

8. Conselho Federal de Enfermagem (BR). Resolução COFEN197/1997. Rio de Janeiro: COFEN; 1997.

9. Silva MBGM, Tonelli ALN, Lacerda MR. Instrumentos do cuidado humanizado de enfermagem: uma reflexão. Cogitare Enferm 2003; 8(1):59-64.

10. Dela Coleta JAD, Dela Coleta MFD. Escalas para medida de atitudes e outras variáveis psicossociais. Ribeirão Preto (SP): Escola de Enfermagem de Ribeirão Preto, Universidade de São Paulo; 1996.

11. Silva LF, Damasceno MMC, Carvalho CML, Souza PDS. Cuidado de enfermagem: o sentido para enfermeiros e pacientes. Rev Bras Enferm 2001; 54(4): 578-88. 